\title{
Donut-Like Topology of Synaptic Vesicles with a Central Cluster of Mitochondria Wrapped into Membrane Protrusions: A Novel Structure-Function Module of the Adult Calyx of Held
}

\author{
Verena C. Wimmer, Heinz Horstmann, Alexander Groh, and Thomas Kuner \\ Abteilung Zellphysiologie, Max-Planck-Institut für medizinische Forschung, 69120 Heidelberg, Germany
}

\begin{abstract}
Structural and functional properties of synapses are intricately and reciprocally coupled. To cope with the functional requirements in auditory processing, the calyx of Held developed distinct structural specializations such as a large number of active zones, large size, elaborate morphology, and defined distribution of ion channels. These specializations typically appear during postnatal maturation within the first 3 weeks of life and are accompanied by marked changes in the properties of synaptic transmission. We examined the arrangement of synaptic vesicles at different postnatal stages of maturation by genetically labeling vesicles with the fluorescent fusion protein synaptophysin-enhanced green fluorescent protein. Fluorescence and electron microscopy-based analyses revealed a new anatomical specialization in the mature calyx of Held. Within small, membrane-delimited compartments (swellings), synaptic vesicles formed donut-like assemblies around a central cluster of interconnected mitochondria. Adult calyces contained $\sim 100$ such structural units, each of them consisting of $\sim 800$ synaptic vesicles, six to nine mitochondria, and five to nine active zones. A donut of synaptic vesicles measured $\sim 1 \mu \mathrm{m}$ in diameter and was placed in a swelling with a volume of $\sim 5 \mathrm{fl}$. Conspicuously, this structural specialization appears with the onset of hearing and may contribute to maturational changes in presynaptic function.
\end{abstract}

Key words: synaptic transmission; mitochondrial function; synaptic vesicle topology; 3D reconstruction; presynaptic mechanisms; viral gene transfer

\section{Introduction}

The neuronal circuitry of the auditory system has developed unique structural specializations to meet the demanding functional requirements needed for the processing of sound (Rouiller et al., 1986; Reuss, 2000). The localization of sound sources, for example, requires highly precise, phase-locked, high-frequency transmission of signals along the auditory pathway (McAlpine, 2005). Neurons of the ventral cochlear nucleus (VCN) transmit such signals through a giant nerve terminal known as the calyx of Held to the principal neurons of the contralateral medial nucleus of the trapezoid body (MNTB) (for review, see von Gersdorff and Borst, 2002). To function as a reliable relay synapse, the calyx of Held developed distinct structural specializations. Compared with a typical central terminal containing a single active zone (AZ), the calyx contains 500-800 AZs functioning in parallel (Sätzler et al., 2002). Such a large number of active zones could

Received Aug. 4, 2005; revised Nov. 8, 2005; accepted Nov. 8, 2005.

This work was supported by the Max-Planck-Gesellschaft, Gertrud-Reemtsma-Promovendenpreis (A.G.), and Claussen-Simon-Stiftung (T.K.). We thank Bert Sakmann for generous support and critical input, Kurt Sätzler for advice on reconstructions, and Pavel Osten and Alexander Kolleker for providing a Sindbis vector for VGlut1-EGFP and a lentivirus expressing EGFP.

Correspondence should be addressed to Thomas Kuner, Abteilung Zellphysiologie, Max-Planck-Institut für medizinische Forschung, Jahnstrasse 29, 69120 Heidelberg, Germany. E-mail: kuner@mpimf-heidelberg.mpg.de. DOI:10.1523/JNEUROSCI.3268-05.2006

Copyright $\odot 2006$ Society for Neuroscience $\quad$ 0270-6474/06/260109-08\$15.00/0 only be accommodated by an increase in size, making the calyx one of the largest nerve terminals in the CNS of mammals. Furthermore, the organization of the mature calyx into small units of volume, so-called stalks and swellings (Rowland et al., 2000), is thought to facilitate clearance of neurotransmitter from the synaptic cleft and thereby support high-frequency transmission. Also on the level of protein distribution, the architecture of the calyx is designed to meet specific functional requirements: the restricted localization of $\mathrm{Na}$ channels to proximal parts of the calyx results in narrow action potential waveforms ensuring reliable, high-frequency transmission (Leao et al., 2005).

Structural and functional specializations of the calyx emerge during the first 3 postnatal weeks. At postnatal day 5 (P5), calyces are club-shaped and extend several thin filopodia, whereas they form a highly digitated structure in the mature state at around P21 (Morest, 1968; Kandler and Friauf, 1993). These morphological changes are accompanied by distinct changes in the properties of synaptic transmission (von Gersdorff and Borst, 2002). Synapses at P5-P8 exhibit strong short-term synaptic depression (STD) and have high release probabilities, wide action potentials, and several other distinct features. In contrast, after the onset of hearing, mature synapses show much less STD, low release probabilities, and narrow action potentials (Taschenberger and von Gersdorff, 2000; Taschenberger et al., 2002). Hence, from the multiplicity of AZs to morphological specializations and distinct 
distribution of $\mathrm{Na}$ channels, structural properties are tightly linked to function in the calyx of Held. In this context, it is interesting to ask whether cellular organelles undergo distinct morphological rearrangements during postnatal maturation as well, and how such structural factors could contribute to the functional maturation of the calyx. This is particularly relevant with regard to synaptic vesicles (SVs), because a continuous replenishment of SVs at the AZs is required to sustain high-frequency neurotransmission.

SVs were visualized by tagging them with synaptophysin-enhanced green fluorescent protein (synaptophysin-EGFP), selectively expressed in VCN projection neurons by viral gene transfer and stereotaxic delivery. We found that during postnatal maturation, SVs became organized into distinct ring-like assemblies, which first occur at P12 with the onset of hearing and are fully mature after P21. These SV donuts are mainly located within specialized structural compartments at the periphery of the calyx, possibly constituting functionally independent units. On the ultrastructural level, they consist of central mitochondrial clusters surrounded by several AZs, constituting a new topological specialization.

\section{Materials and Methods}

Constructs. EGFP and synaptophysin-EGFP were subcloned into $\alpha$-viral vectors (Wimmer et al., 2004). A construct encoding monomeric red fluorescent protein (mRFP1) and synaptotagmin I-pHluorin linked by the 2A peptide (de Felipe et al., 1999) (APVKQTLNFDLLKLAGDVESNPGP) was subcloned with PCR-based methods into pSinRep5lacZ (Invitrogen, San Diego, CA). Superecliptic pHluorin fused with synaptotagmin and mRFP1 were kindly provided by $\mathrm{T}$. Ryan (Cornell University, New York, NY) and R. Tsien (University of California, San Diego, San Diego, CA), respectively.

Viral gene transfer and stereotaxic injections. The production of recombinant $\alpha$ viruses (Sindbis and Semliki Forest virus), stereotaxic injections into the $\mathrm{VCN}$, and tissue processing were done as reported previously (Wimmer et al., 2004). Briefly, the VCN of Sprague Dawley rats at P7P21 was injected with $\sim 1 \mu$ l of virus solution. After in vivo protein expression for $24-36 \mathrm{~h}$, rats were perfused transcardially with PBS containing 4\% paraformaldehyde (PFA). Coronal sections of the auditory brain stem (thickness, 22-100 $\mu \mathrm{m}$ ) were cut on a vibratome and examined with confocal microscopy. All experiments were performed according to biosafety level 2 guidelines specified in the German GenTSV and animal welfare guidelines specified in the TierSchG.

Visualization of mitochondria and immunohistochemistry. Mitochondria were visualized in $50-\mu \mathrm{m}$-thick fixed brain slices by overnight incubation in PBS containing streptavidin-Alexa594 (Invitrogen) at a final concentration of $2 \mu \mathrm{g} / \mathrm{ml}$ in PBS $/ 0.1 \%$ Triton X-100 and $0.1 \%$ Triton $\mathrm{X}-100$. Subsequently, sections were washed in PBS and mounted on glass slides using SlowFade Light mounting medium (Invitrogen).

For immunofluorescence, $500-\mu \mathrm{m}$-thick PFA-fixed slices were cryoprotected with $30 \%$ dimethyl-formamide. A tissue block measuring $1.2 \times 1.2 \mathrm{~mm}$ was frozen in a drop of Tissue Freezing Medium (Jung, Nussloch, Germany) and cryocut into 22- $\mu$ m-thick sections using an ultramicrotome (Ultracut S; Leica, Nussloch, Germany). Synaptophysin was detected using an anti-synaptophysin antibody (reference 101002; Synaptic Systems, Gottingen, Germany). Sections were floated on drops of incubation media. Slices were stained with primary antibody (1:50) for $90 \mathrm{~min}$ at room temperature. Subsequently, sections were washed and incubated with secondary anti-rabbit antibody for $30 \mathrm{~min}$ (1:150; AlexaFluor488; Invitrogen). Finally, the slices were washed again and mounted in Vectashield [4',6'-diamidino-2-phenylindole (DAPI)] (Vector Laboratories, Orton Southgate, Peterborough, UK).

Confocal laser scanning fluorescence microscopy and image analysis. Confocal image stacks of PFA-fixed brain slices were acquired with a Leica TCS SP2 laser scanning microscope with $350 \mathrm{~nm}$ (DAPI), $488 \mathrm{~nm}$ (EGFP), $543 \mathrm{~nm}$ (mRFP1), or $568 \mathrm{~nm}$ (Alexa594) excitation wavelength using 10,40 , or $63 \times$ Leica objectives. High-resolution three-dimensional
(3D) stacks were recorded using $63 \times$ oil or glycerol objectives. Stacks were oversampled in the $Z$ direction by a factor of 1.5 and were subsequently deconvolved using Huygens2 software (version 2.31; Scientific Volume Imaging, Hilversum, The Netherlands). 3D reconstructions were generated using Volocity2 (Improvision, Lexington, MA). Images shown here are snapshots of reconstructions generated in the $3 \mathrm{D}$ viewer. Classifier, a software extension of Volocity, was used to identify and render calyx synapses and SV clusters, respectively. Data sets containing information about size, volume, and localization of calyces and SV clusters within a calyx synapse were exported to a Microsoft (Redmond, WA) Excel spreadsheet for further analysis.

Estimations of volumes and surface areas from 3D reconstructions of fluorescence data are associated with errors arising from limited resolution in the $z$-axis and limits in rendering accuracy. The $z$-axis error was estimated by scanning fluorescent microspheres $(0.2 \mu \mathrm{m}$; Invitrogen $)$ with settings identical to those used when imaging calyces. The shape of the beads was skewed in the $Z$ direction by a factor of two. Therefore, we overestimate the calyx volume approximately twofold. The surface area of an object can increase strongly while keeping the volume quite constant, for example by forming infoldings. Because these can be below the $X-Y$ resolution limit of fluorescence microscopy, errors in surface area estimations can be large and difficult to predict.

Electron microscopy. A P22 Sprague Dawley rat was used to generate a partial ultrastructural reconstruction of the calyx. The animal was anesthetized with sodium pentobarbital and perfused transcardially with 10 $\mathrm{ml}$ of PBS followed by $15 \mathrm{ml}$ of a solution containing $4 \%$ PFA and $2.5 \%$ glutaraldehyde. After extraction of the brain and overnight postfixation in the same solution, the brainstem was cut into $200-\mu \mathrm{m}$-thick coronal sections with a vibratome. A tissue block containing the MNTB was excised and washed with $0.1 \mathrm{M}$ PBS and subsequently postfixed with $2 \%$ $\mathrm{OsO}_{4} / 1.5 \%$ potassium-hexa-cyano-ferrate in water. After washing for $3 \mathrm{~h}$ in water, the sections were incubated overnight in $0.4 \%$ uranylacetate in water at $4^{\circ} \mathrm{C}$. Then, the tissue was dehydrated by incubation in ascending concentrations of ethanol and propylene oxide. Each section was embedded in Spurr and polymerized at $70^{\circ}$ C. Serial sections $(70 \mathrm{~nm}$ thick) were cut from the MNTB using a Leica Ultracut $S$ vibratome (diamond knife) and collected on Formvar-coated copper grids. For contrast enhancement, sections were treated with Reynold's Pb-citrate and uralylacetate for $25 \mathrm{~min}$ in the dark. Electron micrographs were obtained using a Philips 400T microscope.

$3 D$ reconstruction of serial electron micrographs. Three-dimensional reconstructions were made by alignment of serial EM images using the software CAR (Sätzler et al., 2002). Successive sections were aligned to each other by rotation and translation until corresponding structures of adjacent sections superimposed. Subsequently, subcellular structures were manually contoured, 3D surface reconstructions generated, and volumes calculated by CAR.

\section{Results}

\section{Spatial organization of synaptic vesicles during postnatal maturation of the calyx of Held}

SVs of the rat calyx of Held were fluorescently labeled by expressing synaptophysin-EGFP in projection neurons of the ventral cochlear nucleus. Figure 1 shows the arrangement of synaptic vesicles at different postnatal stages of maturation. An immature calyx at P11 has a typical club-like shape well known from volume renderings of the calyx by serial EM sectioning, expression of EGFP, or introduction of fluorescent dyes (Borst et al., 1995; Sätzler et al., 2002; Wimmer et al., 2004). The SVs were almost homogenously distributed and showed a slight tendency to form aggregates (Fig. 1A). Concomitant with the onset of hearing at P12-P14 (Blatchley et al., 1987), when the calyx has developed a more digitated morphology, ring-like local accumulations of SVs became apparent (Fig. $1 B$ ). This process of organizing SVs into distinct geometrical units ended around P21, when most SVs were arranged in clusters resembling donuts (Fig. 1C). The detailed structure of donuts is shown in Figure $1 D-G$; additional 

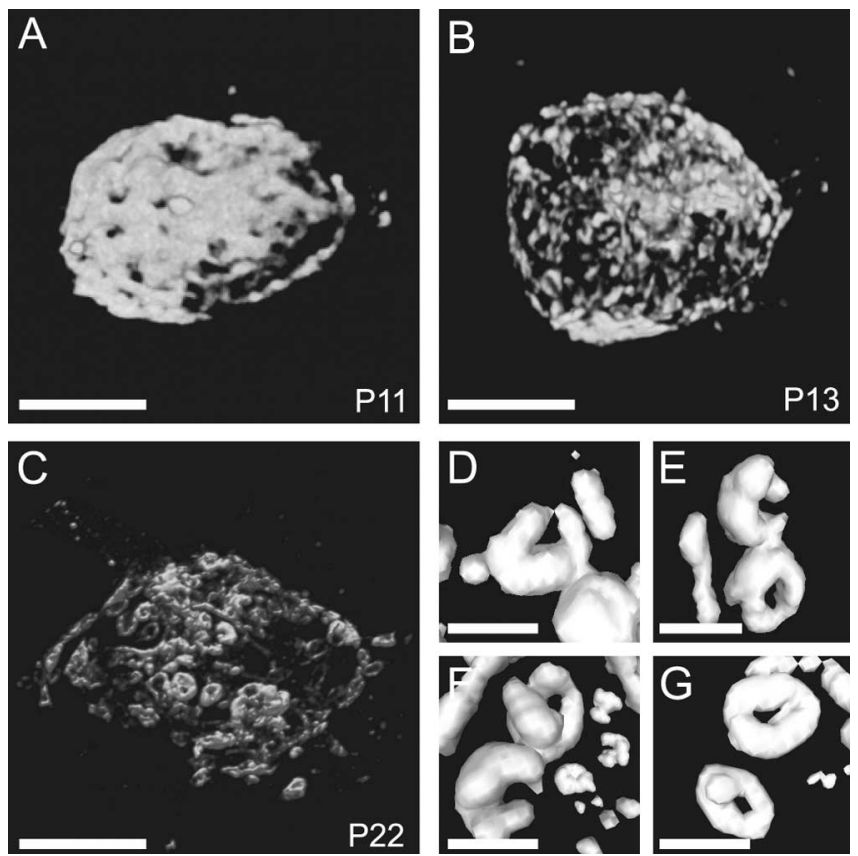

Figure 1. Organization of SV into donut-shaped clusters during postnatal maturation. Examples of synaptophysin-EGFP-labeled SVs in calyces at different postnatal stages are shown. Projections of 3D-reconstructed confocal image stacks are shown. Each panel shows a representative example of two or three independent experiments. $A, S V$ s predominantly arranged in large continuous heaps at P11. B, Clustering of SVs at P13. C, Ring-like SV clusters (donuts) at P22. D-G, High magnification of surface-rendered $P 22$ donuts. Scale bars: $A-C, 5 \mu \mathrm{m} ; D-G, 1 \mu \mathrm{m}$.

examples and a 3D representation are included in the supplemental material (Fig. S1 and Movie S1, available at www. jneurosci.org). Donuts had outer diameters of $\sim 1 \mu \mathrm{m}$ with a hollow center of $\sim 0.5 \mu \mathrm{m}$ in diameter. Hence, during postnatal maturation, with the onset of hearing, SVs redistribute into highly ordered, ring-like assemblies. It may seem surprising that this structural unit had not been discovered previously, but most previous studies focused on immature calyces and did not use 3D reconstructions to analyze immunohistochemical data.

\section{Donuts exist independent of}

synaptophysin-EGFP overexpression

Overexpression of a protein or its fusion with GFP or other protein domains can affect proper maturation, delivery, and subcellular targeting (Szebenyi et al., 2002; Lisenbee et al., 2003). To test whether SV donuts were caused by viral overexpression, we immunostained untreated MNTBs with an antibody directed against synaptophysin and prepared 3D reconstructions from image stacks generated with confocal microscopy. Figure $2 \mathrm{~A}$ shows several calyx terminals surrounding their postsynaptic principal MNTB neurons, the nuclei of which were stained with DAPI. Even in the single optical section shown here, donuts can be readily identified when appropriately oriented in the imaging plane. Clusters of SVs were evident and could be directly demonstrated in 3D reconstructions (Fig. $2 \mathrm{~B}$ ). The immunostained SV donuts had the same dimensions and occurred with the same frequency as in synaptophysin-EGFP-expressing terminals. One could still argue that the donuts are a structural peculiarity of synaptophysin. However, the overexpression of GFP-tagged vesicular glutamate transporter VGluT1 and synaptotagmin I also revealed SV donuts very similar to those described above (Fig. $2 C, D)$. Thus, we conclude that donut-like SV clusters are a specific geometric property of adult calyces.
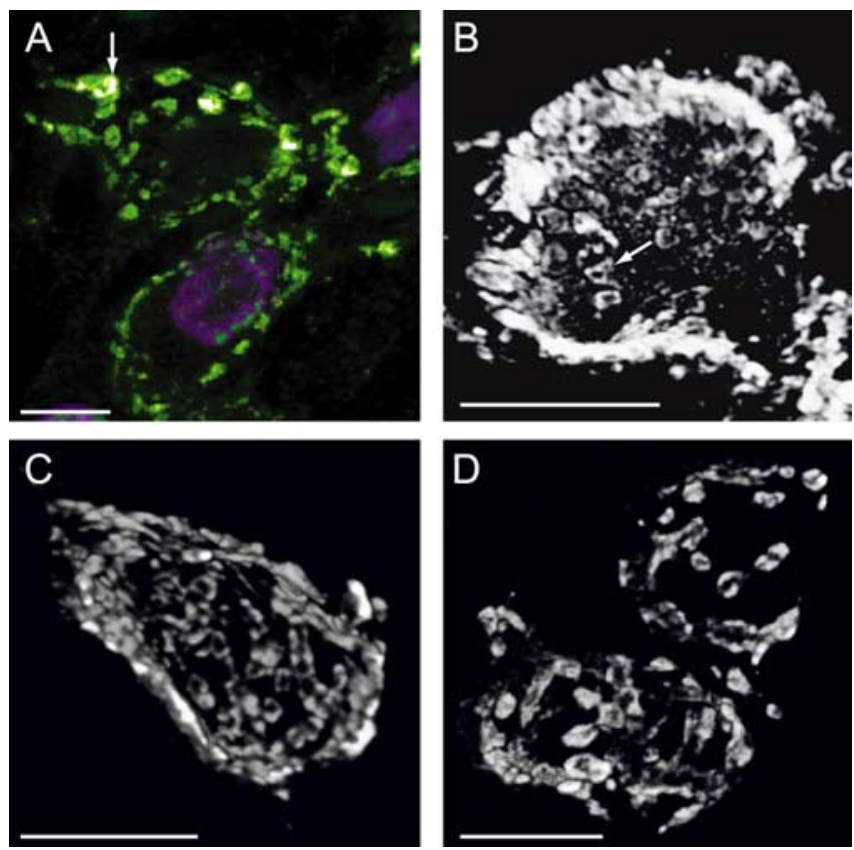

Figure 2. Ring-like SV clusters are present in untreated tissue and after expression of other SV proteins. $\boldsymbol{A}, \boldsymbol{B}$, SV s were stained with an anti-synaptophysin antibody in fixed brain slices generated from a P22 rat. $\boldsymbol{A}$, Representative single confocal image frame. SVs are shown in green; the nucleus of the postsynaptic soma was visualized using DAPI (violet). $\boldsymbol{B}, 3 \mathrm{D}$ reconstruction of a confocal stack showing SV donuts (arrow). C, 3D reconstruction of a confocal stack illustrating SV donuts labeled with VGluT1-EGFP. $\boldsymbol{D}$, As is $\boldsymbol{C}$, but donuts visualized by synaptotagmin-pHluorin expression. The image shows SV clusters of two adjacent calyces. Scale bars, $5 \mu \mathrm{m}$.

\section{Number of donut-like SV clusters increases whereas total} vesicle volume remains constant during postnatal maturation The number and volume of SV clusters was determined in immature and mature calyces using an automatized object classifier. Figure 3, $A$ and $B$, shows renderings of distinct contiguous volumes in a P11 and P22 terminal, respectively. The original data set shown in gray was exactly modeled by the rendering, suggesting an accurate representation of the total vesicle space by the classification algorithm applied. The average volume of the total vesicle space at $\mathrm{P} 11$ was $328 \pm 146 \mu \mathrm{m}^{3}(n=4$; mean $\pm \mathrm{SD})$, and the average volume of individual clusters was $21 \pm 12 \mu \mathrm{m}^{3}$, suggesting a total number of $17 \pm 3 \mathrm{SV}$ clusters. The size histograms of SV clusters in juvenile synapses, however, were clearly skewed to very few (usually one) but large clusters plus several small SV accumulations (data not shown).

SV clusters of P22 terminals could be accurately isolated (Fig. $3 B)$. The total vesicle space was $211 \pm 41 \mu \mathrm{m}^{3}(n=5)$ with an average cluster volume of $2.4 \pm 0.8 \mu \mathrm{m}^{3}$, yielding $93 \pm 26 \mathrm{SV}$ clusters. P13 terminals contained $101 \pm 20(n=3)$ SV clusters (Fig. $3 C$ ). Size histograms revealed a rather homogenous cluster size in adult calyces (data not shown). Hence, the total volume of SV space remained unchanged, whereas the average cluster volume decreased, and therefore, the number of SV clusters increased significantly.

\section{Compartmentalization and formation of SV donuts}

The maturational formation of SV donuts coincided with the known compartmentalization of the calyx, implying the question of how SV distribution and presynaptic morphology superimpose. To address this question, we first re-examined maturational changes in the calyx morphology. Figure $4 A-D$ shows $3 D$ renderings of calyces at P8, P13, P22, and P28, confirming a subdivision 
of presynaptic space into progressively smaller units while maintaining the overall volume of the terminal. This result was further substantiated by determining the calyx volume and surface area at different postnatal ages, revealing an average volume of $\sim 1 \mathrm{pl}$ at all stages investigated (Fig. $4 E)$ but a twofold increase in surface area from P8 to P22 (Fig. 4F). The maximal surface area was reached at around P12, concurrent with the onset of hearing, and declined thereafter to the adult level. Hence, the morphological specialization was still incomplete at the onset of hearing and continued at least until P28, although with less pronounced changes occurring after P21.

Next, we examined where SV donuts are located in the presynaptic volume. Figure $5 A$ shows a $3 \mathrm{D}$ surface rendering of a P23 calyx with SVs labeled with pHluorin-synaptotagmin I and the calyx filled by coexpression of cytosolic mRFP1. Clearly, synaptic vesicles are located in the peripheral compartments, mostly sparing the central volume of the stalks and initial axon segment. This finding is emphasized in Figure 5B, highlighting the overlapping regions in yellow. The predominant localization of SVs close to the plasma membrane is further illustrated in single-frame cross sections shown in Figure 5, $C$ and $D$. In contrast, SVs occupied the entire available volume in an immature P10 calyx (Fig. 5E). Independent of the postnatal stage, only a few SV clusters were found in the axonal compartment (Fig. $5 A, B, E$ ). Thus, in mature calyces, SV clusters are predominantly located in distinct compartments, referred to as swellings (Rowland et al., 2000), in close apposition to the presynaptic plasma membrane. Furthermore, SV clusters appear as the presynaptic plasma membrane begins to form swellings at the onset of hearing.

\section{Ultrastructural correlate of SV donuts}

To elucidate the organizing principle underlying SV donuts at the ultrastructural level, we reconstructed individual P22 swellings using serial electron microscopic images. Figure $6 A$ shows an electron micrograph of a calyx swelling with some of the membrane structures traced. The $3 \mathrm{D}$ reconstruction based on 70 consecutive sections shows four swelling-like membrane compartments, two of which were interconnected by a thin cytoplasmic bridge (Fig. $6 B$, arrow). The ultrastructural equivalent of a donut emerged after digitally removing the mitochondria and AZs from the reconstruction (Fig. 6C,D). When the size of SVs was artificially increased to match the resolution of light microscopy, a donut shape was readily identified (Fig. 6E). The dimensions of the donut were identical to those determined with fluorescently labeled SVs (external diameter, $\sim 1 \mu \mathrm{m}$ ). The donut shown in Figure $6 E$ consisted of 810 SVs. A stereo image illustrates the flat morphology of the swelling, distribution of SVs, and close contact of mitochondria with the plasma membrane in the central region of the donut (Fig. $6 F$ ).

The central part of the donut was occupied by a cluster of mitochondria (Fig. 6C) forming large tubular structures (Fig. $6 G$ ). Each swelling contained six to nine mitochondria, of which four or five were interconnected. This arrangement could reflect individual mitochondria connected with each other (Brodin et al., 1999; Skulachev, 2001) or may result from the segmentation of a single mitochondrion (King et al., 1996; Rowland et al., 2000). Because the formation of tubular structures could be
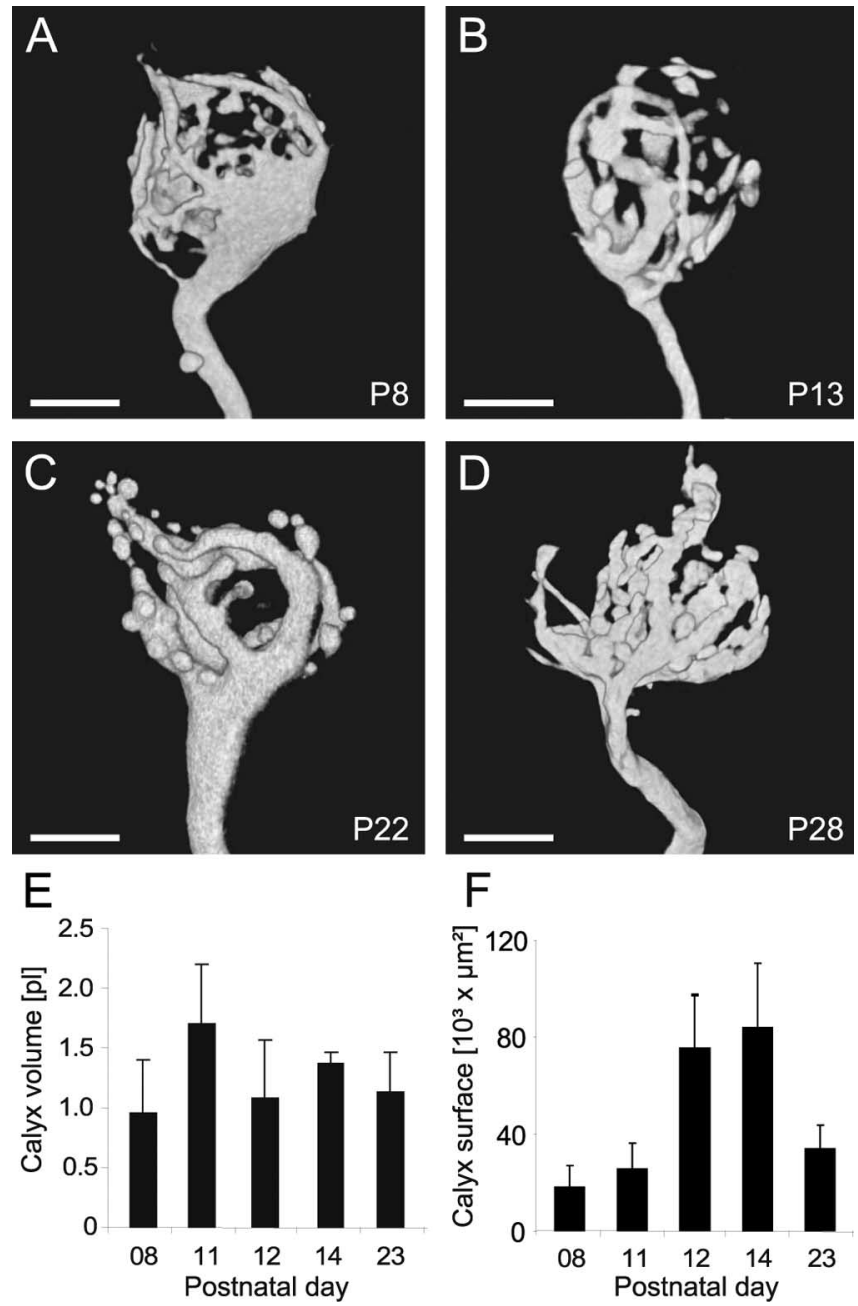

Figure 4. Emergence of donuts parallels morphological maturation of the calyx. Examples of EGFP-expressing calyces at different developmental stages are shown. Confocal stacks were deconvolved and 3D reconstructed. Representative images of 5-10 calyces obtained in two or three independent experiments for each age group. $A, A t P 8$, calyces consist of one large compartment and short, thin semidifferentiated stalks. $\boldsymbol{B}$, Four to six long thick stalks develop until P13. C, Numerous swellings with thin connecting necks line each stalk at P22. D, Until P28, the number of swellings and the overall complexity of the calyx further increases. Scale bars, $5 \mu \mathrm{m}$. $\boldsymbol{E}$, Average volume of EGFP-filled calyces derived from $3 D$ reconstructions of high-resolution confocal stacks (mean \pm SD; $n=3-7 ;$ no significant difference, ANOVA). The volumes are not corrected for possible deviations attributable to limited z-axis resolution (see Materials and Methods and Discussion). $\boldsymbol{F}$, As in $\boldsymbol{E}$, but average surface area (mean \pm SD; P8, P12/P14, and P22 are significantly different, ANOVA). 

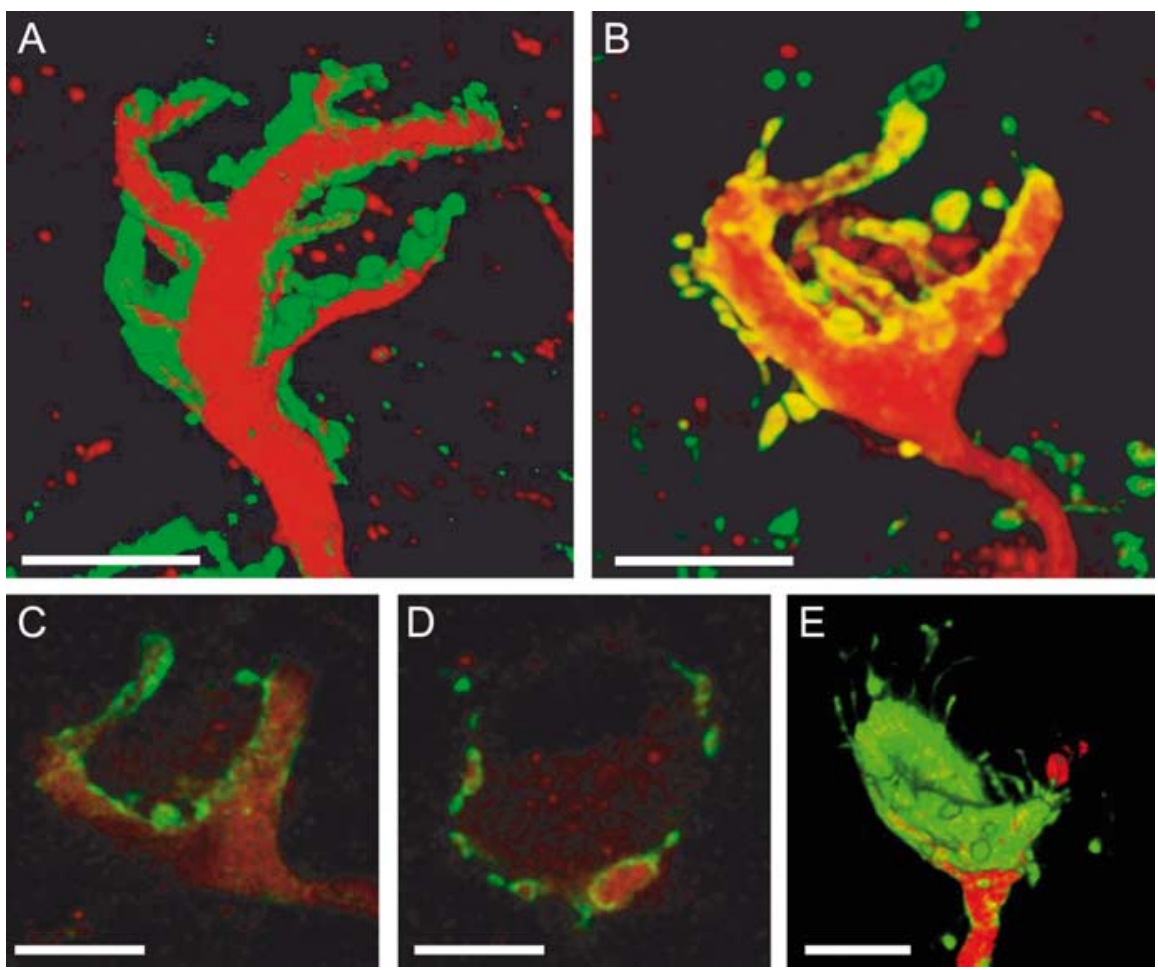

Figure 5. SVs localize to swellings. Simultaneous expression of pHluorin-synaptotagmin I and mRFP1 in P22 calyces. Images of two calyces obtained from a single experiment. $\boldsymbol{A}$, Nontransparent surface rendering of SVs and calyx volume. $\boldsymbol{B}$, Transparent overlay of SVs and calyx volume. $\boldsymbol{C}, \boldsymbol{D}$, Single image frames of the confocal stack shown in $\boldsymbol{B}$. $\boldsymbol{E}$, As in $\boldsymbol{B}$, but showing a P10 calyx. Green, SVs; red, volume fill; yellow, overlay of SVs and calyx volume. Scale bars, $5 \mu \mathrm{m}$.

linked to enhanced mitochondrial function, we examined whether mitochondrial clustering was different in immature versus mature calyces. In P8 terminals, mitochondria were distributed with no clear pattern throughout the available volume (Fig. $6 \mathrm{H}$ ) but nevertheless formed tubular networks. When counting the number of complex mitochondria with more than two ends, we found that these comprised $\sim 29 \%$ of all mitochondria in P8, compared with $25 \%$ in P23. Hence, the distribution of mitochondria changed into a donut-centered pattern during postnatal maturation, but the fraction of interconnected mitochondria remained unchanged between P8 and P23 calyces.

Active zones, defined as the presynaptic electron-dense area of a synaptic contact [for details, see Sätzler et al. (2002)], were mainly situated underneath the ring of SVs (Fig. $6 B, C$ ) but still close enough to mitochondria to form adherence complexes (Rowland et al., 2000). As illustrated in Figure 6F, no AZs and only very few SVs are situated below (or above) the central cluster of mitochondria. Typically, each AZ was associated with a heap of SVs, but we also found large SV clusters lacking nearby active zones (Fig. 6B, C, asterisks). Each swelling, and hence each SV donut, contained $6.8 \pm 0.9(n=4)$ active zones.

The plasma membrane was tightly wrapped around the SV donut with its central mitochondrial cluster creating a distinct compartment with a volume of $4.8 \pm 0.9 \mathrm{fl}$. This compartment was connected to the stalks of the terminal through thin necks. Because there was only one such neck present in the EM 3D reconstruction, we analyzed the neck diameters in fluorescence $3 \mathrm{D}$ reconstructions such as those shown in Figure 4 . We found an average neck diameter of $0.7 \pm 0.08 \mu \mathrm{m}(n=15)$ and about the same length of the neck. Typical swellings measured $\sim 2-3 \mu \mathrm{m}$ in diameter; stalks were in the range of $2-8 \mu \mathrm{m}$. Hence, the constriction formed between swellings and stalks could provide a diffusion barrier, possibly creating a distinct biochemical compartment.

In summary, SV donuts are located in swellings and contain a central cluster of interconnected mitochondria, constituting a new structural principle of organizing key cellular organelles.

\section{Distribution of mitochondria}

Because the EM analysis necessarily focused on a few donut structures, we examined the distribution of mitochondria and SVs on the level of light microscopy by overexpressing synaptophysin-EGFP and staining mitochondria with streptavidinAlexa dye (Kirkeby et al., 1993). Figure 7A shows mitochondrial clusters (red) surrounded by SV clusters (green). A cross section through an individual calyx further illustrates the concept that mitochondrial clusters are surrounded by SVs (Fig. $7 B$, arrow). Hence, SVs form ring-like assemblies with central clusters of mitochondria, situated in distinct membrane compartments of mature calyces.

\section{Discussion}

This study describes a new topological property of a central presynaptic nerve terminal in the adult nervous system of the rat: within distinct membrane protrusions, synaptic vesicles form donut-like assemblies housing a central cluster of interconnected mitochondria (Fig. 8). Because this structural specialization forms with the onset of hearing, it may contribute to the changes in functional properties known to accompany postnatal calyx maturation.

\section{Donut-like vesicle assemblies appear with the onset of hearing} Overexpressing synaptophysin-EGFP in the calyx of Held, we found that SVs are homogeneously distributed in immature terminals before the onset of hearing but started to be predominantly arranged in donut-like assemblies thereafter (Fig. 8). This topology is not induced by overexpression of synaptophysinEGFP, because other SV proteins and immunostains with synaptophysin antibodies also revealed identical donut morphologies and numbers. We suggest that SV donuts predominantly represent the reserve pool (Rizzoli and Betz, 2005), also referred to as the resting pool (de Lange et al., 2003), of SVs, which makes up $\sim 85-95 \%$ of the total pool. To our knowledge, ring-like SV assemblies were so far only described in the neuromuscular nerve endings of Drosophila, where they reflect the cycling pool of SVs, with the reserve pool located in the center of the ring (Kuromi and Kidokoro, 1998). Hence, apart from their similar appearance, the ring-like assemblies of SVs differ fundamentally in these two synapses.

\section{A central cluster of interconnected mitochondria}

Reconstructions of serial EM sections revealed that the apparent central void space of SV donuts is occupied by a cluster of mitochondria. These mitochondria were tightly packed and interconnected. Distinct subcellular localization and interconnection of mitochondria have been described in various cell types including neurons and may allow functional coupling (King et al., 1996; 
Skulachev, 2001; Hollenbeck, 2005). Boosting mitochondrial function by such coupling could affect several processes essential for synaptic transmission.

More efficient ATP production would directly support the function of the nearby SV cycle, because many essential reactions such as priming, transport, and filling of glutamate into SVs depend on ATP (Sudhof, 1995; Hollenbeck, 2005). Maintaining a high concentration of glutamate close to SVs will enhance refilling of recycling SVs with neurotransmitter. Extrusion of $\mathrm{Ca}^{2+}$ is necessary to maintain efficient phasic $\mathrm{Ca}^{2+}$-dependent neurotransmitter release. In this regard, mitochondria may play a role at $\mathrm{Ca}^{2+}$ concentrations exceeding $2.5 \mu \mathrm{M}$, whereas $\mathrm{Ca}^{2+}$ extrusion below $2.5 \mu \mathrm{M}$ is predominantly accomplished by $\mathrm{Na}^{+} / \mathrm{Ca}^{2+}$ exchangers at the plasma membrane (Billups and Forsythe, 2002; Kim et al., 2005). Furthermore, Kim et al. (2005) suggest that developmental changes in $\mathrm{Ca}^{2+}$ buffer expression favor mitochondrial $\mathrm{Ca}^{2+}$ clearance in mature calyces.

Together, these mechanisms are essential to sustain high-frequency synaptic transmission. Consistent with this idea, contacts between mitochondria have been observed previously in the lamprey sensory dorsal column terminals (Brodin et al., 1999), suggesting that the structural principle described here might be of more general relevance.

\section{Sequestration into distinct}

membrane-delimited compartments

The formation of mitochondrial clusters surrounded by SV s coincides with the progressive compartmentalization of the calyx. Beginning with a club-shaped compartment at P8, the calyx differentiates into a complex morphology consisting of multiple stalks and swellings. After hearing onset, the number of small compartments referred to as swellings increased until P22. SV donuts were mainly located to the periphery of the terminal, presumably within swellings emanating from the main stalks. SV accumulations within the stalk lined the plasma membrane apposing the principal neuron (Fig. 5A). Therefore, three processes coincide during postnatal maturation to form the highly specialized adult morphology: compartmentalization, mitochondrial clustering, and formation of SV donuts. We refer to this morphology as the "wrapped donut" (Fig. 8).

\section{Quantitative comparisons and predictions}

SV donuts had outer diameters of 1-2 $\mu \mathrm{m}$ and inner diameters of $0.3-0.5 \mu \mathrm{m}$. They were located inside swellings with volumes of 2-7 fl. The main part of the swelling was separated from the stalks
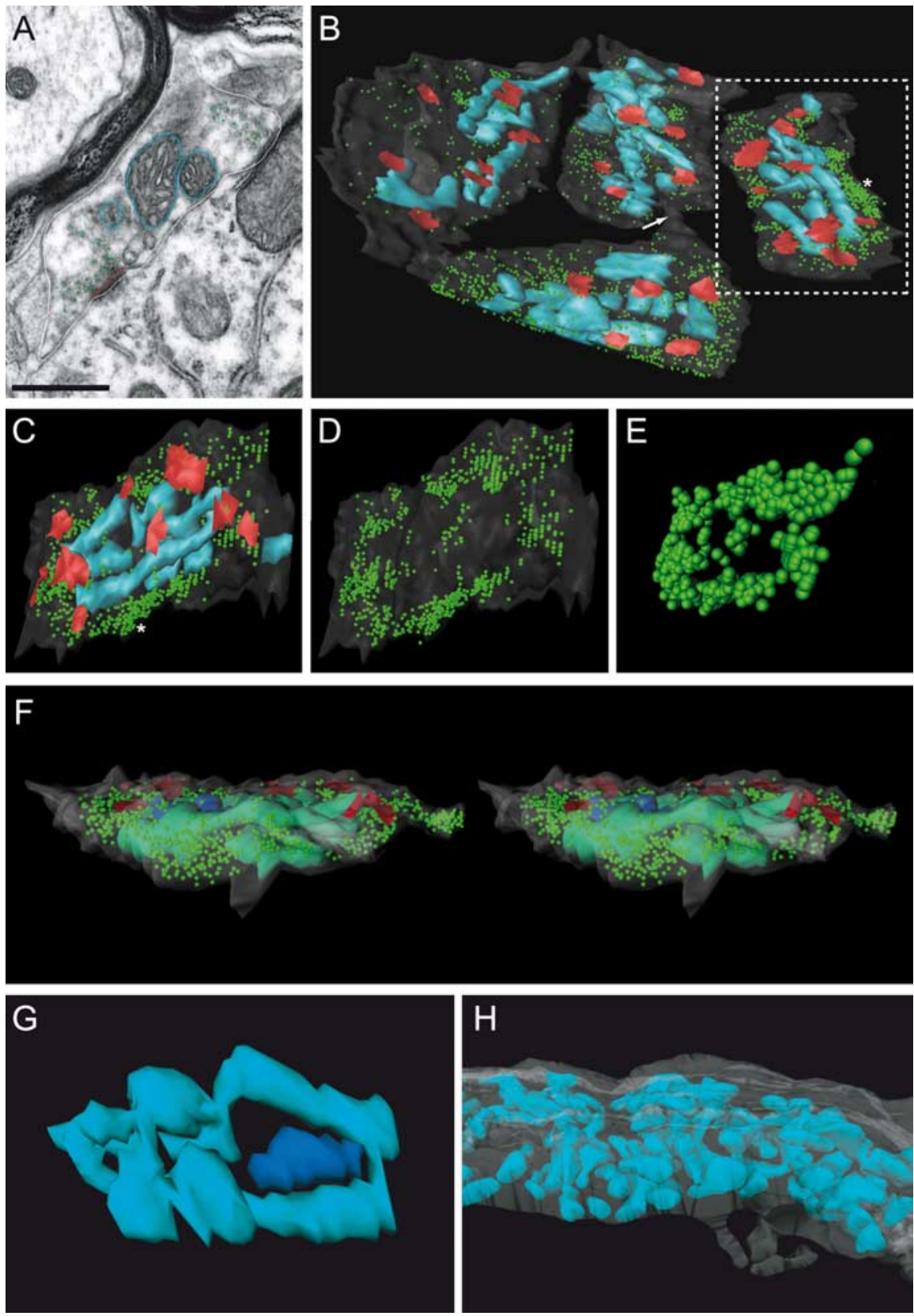

Figure 6. Ultrastructural analysis reveals cluster of interconnected mitochondria in center of SV donuts. 3D reconstruction of 70 serial sections $(70 \mathrm{~nm})$ generated using CAR is shown. $\boldsymbol{A}$, Representative electron micrograph with contoured plasma membrane (gray), mitochondria (cyan), SVs (green), and active zones (red). Scale bar, $500 \mathrm{~nm}$. $\boldsymbol{B}, 3 \mathrm{D}$ reconstruction covering a volume of $\sim 5 \times 5 \times 1 \mu \mathrm{m}$ of a P22 calyx. Four membrane-wrapped compartments comprise central clusters of mitochondria surrounded by SVs. The arrow denotes a cytoplasmatic bridge; accumulations of SVs without a corresponding AZ are indicated with an asterisk. Color code as in $\boldsymbol{A}$. C, Area highlighted in $\boldsymbol{B}$ rotated and slightly magnified. D, Ring-like appearance of SVs. The row-like arrangement of SVs in $\boldsymbol{C}$ and $\boldsymbol{D}$ is caused by the fact that every object identified in $80 \mathrm{~nm}$ serial sections appears at the same $z$ position. $\boldsymbol{E}$, SV s artificially enlarged to the diffraction limit of light microscopy to mimic confocal imaging conditions. $\boldsymbol{F}$, Stereo image of the donut shown in C. G, Magnified view of the mitochondrial cluster shown in C. The cluster consists of approximately six mitochondria, of which five are interconnected (cyan). $\boldsymbol{H}$, Mitochondria are homogeneously distributed but nevertheless formed interconnected clusters at P8 [dataset published by Sätzler et al. (2002)]. 

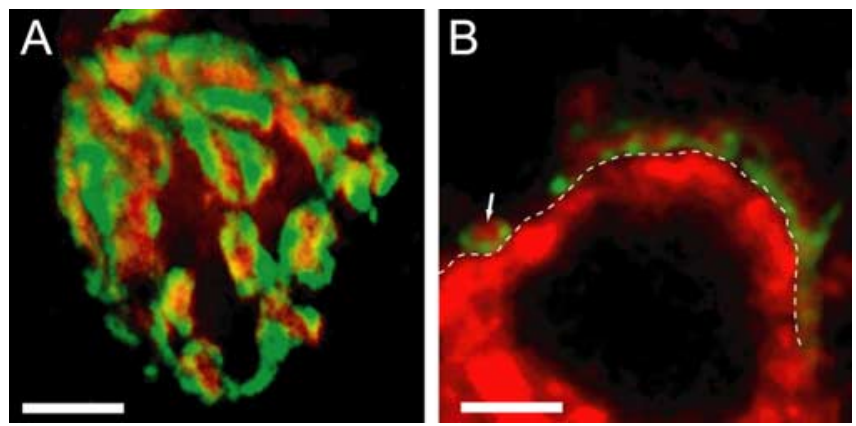

Figure 7. Clustering of mitochondria within SV donuts as a general organizing principle in the adult calyx. Mitochondria were stained with a streptavidin-Alexa594 conjugate in fixed slices containing synaptophysin-EGFP-expressing P22 calyces. A, 3D rendering of a confocal stack. SVs (green) surround mitochondria (red). Note that the blurred background signal stems from stained mitochondria of the principal cell, which contains abundant numbers of mitochondria. $\boldsymbol{B}$, Single confocal frame obtained from a different synapse. In this cross section, the nucleus of the principal neuron generates a void space surrounded by a high density of mitochondria. Within the calyx, the density of mitochondria was lower. The dotted line indicates the border between calyx and principal neuron. Arrow, Well defined cluster of mitochondria. Scale bars, $5 \mu \mathrm{m}$.

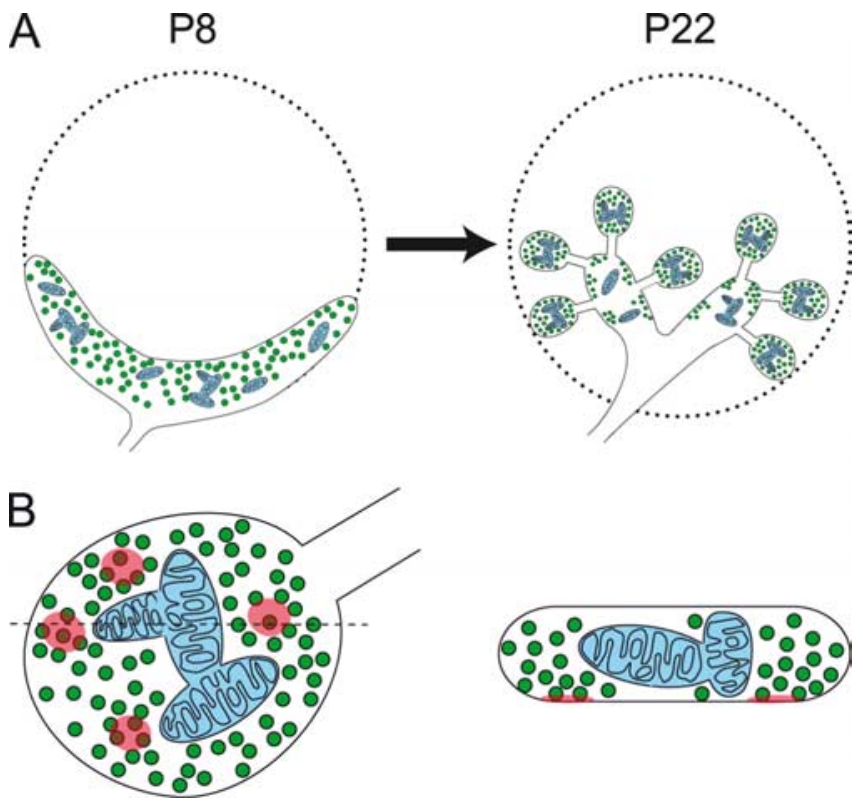

Figure 8. Scheme of wrapped donuts. $A$, With the onset of hearing (arrow), the calyx nerve terminal differentiates from a nonstructured membrane bag into a highly ordered structure consisting of wrapped donuts (SVs surrounding a cluster of mitochondria, both packed into membrane protrusions). $\boldsymbol{B}$, Side view and cross section of a wrapped donut. Green, SVs; blue, mitochondria; red, AZs.

ments. Analogous to the situation found in spines (Svoboda et al., 1996), the neck may not affect much the electrotonic coupling of the swelling to the main stalk, but rather generate a segregated biochemical compartment (Hayashi and Majewska, 2005). Such compartments could entail local control on SV cycling, $\mathrm{Ca}^{2+}$ accumulation and clearance, and regulation of release probability. Because mitochondria have been reported to be highly dynamic and mobile (Polyakov et al., 2003; Muller et al., 2005), their segregation into swellings may effectively trap them in a functionally relevant position.

The total volume of the calyx was estimated from confocal 3D reconstructions of fluorescent calyces to be $\sim 1$ pl, independent of the maturation stage. Because of limitations in spatial resolution in the $z$-axis, we overestimated the true volume about twofold (see Materials and Methods), yielding $\sim 0.5 \mathrm{pl}$ of calyx volume. This number agrees remarkably well with the 0.48 and $0.35-1.05$ pl volumes reported previously (Helmchen et al., 1997; Sätzler et al., 2002). The surface area of the calyx increased about fivefold from P8 to P14 and then settled at a twofold larger surface area at P23 compared with P8. An increase of the surface with constant volume suggests that a fissioning process rather than appositional growth underlies the maturation of the calyx from a club-like structure into its digitated adult morphology (Morest, 1968; Kandler and Friauf, 1993). The transient peak in surface area coinciding with the onset of hearing may suggest that activity-dependent synaptic pruning contributes to the adult connectivity in the MNTB.

Each of the 100 SV clusters was associated with approximately seven AZs, suggesting that $\sim 700 \mathrm{AZs}$ exist in an adult calyx. This number is in close agreement with the 650 AZs reported by Sätzler et al. (2002) for an immature P9 calyx. We counted 810 SVs in the prototypic donut shown in Figure $6 E$, yielding $\sim 115$ SVs per $\mathrm{AZ}$ and a total number of 81000 SVs per calyx. Again, these numbers are consistent with previous estimates (Sätzler et al., 2002; de Lange et al., 2003). Hence, a single swelling stores $~ 800$ quanta of neurotransmitter, of which $\sim 32$ ( $4 \%$ of total SVs) are predicted to be recycling SVs and eight ( $1 \%$ of SVs) to be readily releasable vesicles (Rizzoli and Betz, 2005). The entire calyx could be viewed as an array of 100 independent wrapped donuts, like an axon forming many independent boutons on a single postsynaptic cell.

\section{Organizing principle}

The formation of wrapped donuts, coincident with the onset of hearing, raises the question about mechanisms generating this morphological specialization. One or more as yet unknown molecular factors could be upregulated during development and specifically induce this morphology. However, this would require a concerted action of forming membrane compartments, mitochondrial clusters, and SV donuts simultaneously. Although proteins forming connections between mitochondria have been identified, it seems difficult to imagine a single molecular entity organizing such a complicated series of events. Nevertheless, a clustering of mitochondria might be the first step leading to membrane recruitment and subsequent invagination to generate flat ovoid swellings (Fig. 8). As a matter of geometry, mitochondria will settle in the center of the swellings, leaving the surrounding torus-shaped space for the SVs and other presynaptic organelles such as endosomes.

\section{Contribution to function}

We suggest that wrapped donuts are a specialization of the calyx that supports high-frequency synaptic transmission. This is consistent with the observation that synaptic STD is strong in immature calyces but much less pronounced in mature ones. A mechanistic link between donut topology and STD could be the more efficient generation and trapping of ATP and glutamate close to the site of consumption. The distinct geometry of SV donuts prompts the suggestion that diffusional distances between source and sink of ATP, glutamate, and $\mathrm{Ca}^{2+}$ could be optimized by this arrangement. However, comparing a simulated random distribution of SVs and mitochondria with a donut-like arrangement revealed that both yielded a similar distribution of diffusion distances between mitochondria and SVs (data not shown). Because of the compartmentalization, the concentration of glutamate and ATP could potentially be kept higher, supporting efficient functioning of the synaptic vesicle cycle. 
Mitochondrial oxidative metabolism leads to the local generation of reactive oxygen species, which in turn could link mitochondrial function with cellular functions (Skulachev, 2001). Indeed, presynaptic proteins such as $N$-ethyl-maleimide-sensitive factor have been shown to be inhibited by nitrosylation and hydrogen peroxide (Matsushita et al., 2003, 2005) and therefore might silence transmission after strong metabolic drive. As a result of the flat morphology of the swellings, the central part of the mitochondrial cluster is positioned close to the plasma membrane without intervening AZs or SVs (Fig. $8 \mathrm{~B}$ ). This could be a site of enhanced oxidative activity, thereby facilitating oxygen exchange and avoiding the accumulation of oxygen species at the AZs. Power transmission (Skulachev, 2001) may then maintain ATP delivery at the AZs. Together, these mechanisms support the fidelity of synaptic transmission.

The morphological specializations reported here may also accelerate clearance of glutamate from the synaptic cleft, thereby limiting AMPA receptor desensitization. This is consistent with a strong reduction of desensitization observed during development (Taschenberger et al., 2002). A ring-like arrangement of the AZs within swellings positions the glutamate release sites near the circumference of the swellings, allowing rapid clearance of glutamate from the synaptic cleft by passive diffusion (Renden et al., 2005).

In summary, wrapped donuts are a structure-function unit of the adult calyx of Held emerging around the onset of hearing. The development of $\sim 100$ such independent biochemical compartments may indicate that, after an increase in size to accommodate multiple AZs during adolescence, the adult calyx returns to the principles of bouton-like terminals found elsewhere in the brain.

\section{References}

Billups B, Forsythe ID (2002) Presynaptic mitochondrial calcium sequestration influences transmission at mammalian central synapses. J Neurosci 22:5840-5847.

Blatchley BJ, Cooper WA, Coleman JR (1987) Development of auditory brainstem response to tone pip stimuli in the rat. Brain Res 429:75-84.

Borst JG, Helmchen F, Sakmann B (1995) Pre- and postsynaptic whole-cell recordings in the medial nucleus of the trapezoid body of the rat. J Physiol (Lond) 489:825-840.

Brodin L, Bakeeva L, Shupliakov O (1999) Presynaptic mitochondria and the temporal pattern of neurotransmitter release. Philos Trans R Soc Lond B Biol Sci 354:365-372.

de Felipe P, Martin V, Cortes ML, Ryan M, Izquierdo M (1999) Use of the 2A sequence from foot-and-mouth disease virus in the generation of retroviral vectors for gene therapy. Gene Ther 6:198-208.

de Lange RP, de Roos AD, Borst JG (2003) Two modes of vesicle recycling in the rat calyx of Held. J Neurosci 23:10164-10173.

Fiala JC, Harris KM (1999) Dendritic structure. In: Dendrites (Stuart G, Sprouston N, Häusser M, eds), 1-34. Oxford: Oxford UP.

Hayashi Y, Majewska AK (2005) Dendritic spine geometry: functional implication and regulation. Neuron 46:529-532.

Helmchen F, Borst JG, Sakmann B (1997) Calcium dynamics associated with a single action potential in a CNS presynaptic terminal. Biophys J 72:1458-1471.

Hollenbeck PJ (2005) Mitochondria and neurotransmission: evacuating the synapse. Neuron 47:331-333.

Kandler K, Friauf E (1993) Pre- and postnatal development of efferent connections of the cochlear nucleus in the rat. J Comp Neurol 328:161-184.

Kim MH, Korogod N, Schneggenburger R, Ho WK, Lee SH (2005) Interplay between $\mathrm{Na}^{+} / \mathrm{Ca}^{2+}$ exchangers and mitochondria in $\mathrm{Ca}^{2+}$ clearance at the calyx of Held. J Neurosci 25:6057-6065.

King MJ, Atwood HL, Govind CK (1996) Structural features of crayfish phasic and tonic neuromuscular terminals. J Comp Neurol 372:618-626.
Kirkeby S, Moe D, Bog-Hansen TC, van Noorden CJ (1993) Biotin carboxylases in mitochondria and the cytosol from skeletal and cardiac muscle as detected by avidin binding. Histochemistry 100:415-421.

Kuromi H, Kidokoro Y (1998) Two distinct pools of synaptic vesicles in single presynaptic boutons in a temperature-sensitive Drosophila mutant, shibire. Neuron 20:917-925.

Leao RM, Kushmerick C, Pinaud R, Renden R, Li GL, Taschenberger H, Spirou G, Levinson SR, von Gersdorff H (2005) Presynaptic $\mathrm{Na}^{+}$channels: locus, development, and recovery from inactivation at a high-fidelity synapse. J Neurosci 25:3724-3738.

Lisenbee CS, Karnik SK, Trelease RN (2003) Overexpression and mislocalization of a tail-anchored GFP redefines the identity of peroxisomal ER. Traffic 4:491-501.

Matsushita K, Morrell CN, Cambien B, Yang SX, Yamakuchi M, Bao C, Hara MR, Quick RA, Cao W, O'Rourke B, Lowenstein JM, Pevsner J, Wagner DD, Lowenstein CJ (2003) Nitric oxide regulates exocytosis by $S$-nitrosylation of $N$-ethylmaleimide-sensitive factor. Cell 115:139-150.

Matsushita K, Morrell CN, Mason RJ, Yamakuchi M, Khanday FA, Irani K, Lowenstein CJ (2005) Hydrogen peroxide regulation of endothelial exocytosis by inhibition of $\mathrm{N}$-ethylmaleimide sensitive factor. J Cell Biol 170:73-79.

McAlpine D (2005) Creating a sense of auditory space. J Physiol (Lond) 566:21-28.

Morest DK (1968) The growth of synaptic endings in the mammalian brain: a study of the calyces of the trapezoid body. Z Anat Entwicklungsgesch 127:201-220.

Muller M, Mironov SL, Ivannikov MV, Schmidt J, Richter DW (2005) Mitochondrial organization and motility probed by two-photon microscopy in cultured mouse brainstem neurons. Exp Cell Res 303:114-127.

Polyakov VY, Soukhomlinova MY, Fais D (2003) Fusion, fragmentation, and fission of mitochondria. Biochemistry (Mosc) 68:838-849.

Renden R, Taschenberger H, Puente N, Rusakov DA, Duvoisin R, Wang LY, Lehre KP, von Gersdorff H (2005) Glutamate transporter studies reveal the pruning of metabotropic glutamate receptors and absence of AMPA receptor desensitization at mature calyx of Held synapses. J Neurosci 25:8482-8497.

Reuss S (2000) Introduction to the superior olivary complex. Microsc Res Tech 51:303-306.

Rizzoli SO, Betz WJ (2005) Synaptic vesicle pools. Nat Rev Neurosci 6:57-69.

Rouiller EM, Cronin-Schreiber R, Fekete DM, Ryugo DK (1986) The central projections of intracellularly labeled auditory nerve fibers in cats: an analysis of terminal morphology. J Comp Neurol 249:261-278.

Rowland KC, Irby NK, Spirou GA (2000) Specialized synapse-associated structures within the calyx of Held. J Neurosci 20:9135-9144.

Sätzler K, Sohl LF, Bollmann JH, Borst JG, Frotscher M, Sakmann B, Lubke JH (2002) Three-dimensional reconstruction of a calyx of Held and its postsynaptic principal neuron in the medial nucleus of the trapezoid body. J Neurosci 22:10567-10579.

Skulachev VP (2001) Mitochondrial filaments and clusters as intracellular power-transmitting cables. Trends Biochem Sci 26:23-29.

Sudhof TC (1995) The synaptic vesicle cycle: a cascade of protein-protein interactions. Nature 375:645-653.

Svoboda K, Tank DW, Denk W (1996) Direct measurement of coupling between dendritic spines and shafts. Science 272:716-719.

Szebenyi G, Smith GM, Li P, Brady ST (2002) Overexpression of neurofilament $\mathrm{H}$ disrupts normal cell structure and function. J Neurosci Res 68:185-198.

Taschenberger H, von Gersdorff H (2000) Fine-tuning an auditory synapse for speed and fidelity: developmental changes in presynaptic waveform, EPSC kinetics, and synaptic plasticity. J Neurosci 20:9162-9173.

Taschenberger H, Leao RM, Rowland KC, Spirou GA, von Gersdorff $\mathrm{H}$ (2002) Optimizing synaptic architecture and efficiency for highfrequency transmission. Neuron 36:1127-1143.

von Gersdorff H, Borst JG (2002) Short-term plasticity at the calyx of held. Nat Rev Neurosci 3:53-64.

Wimmer VC, Nevian T, Kuner T (2004) Targeted in vivo expression of proteins in the calyx of Held. Pflügers Arch 449:319-333. 